\title{
MHD Flow of a Viscous Fluid over an Exponentially Stretching Sheet in a Porous Medium
}

\author{
Iftikhar Ahmad, ${ }^{1}$ Muhmmad Sajid, ${ }^{2}$ Wasim Awan, ${ }^{1}$ Muhammad Rafique, ${ }^{3}$ Wajid Aziz, ${ }^{4}$ \\ Manzoor Ahmed, ${ }^{1}$ Aamar Abbasi, ${ }^{1}$ and Moeen Taj ${ }^{1}$ \\ ${ }^{1}$ Department of Mathematics, University of Azad Jammu and Kashmir Muzaffarabad, Azad Kashmir 13100, Pakistan \\ ${ }^{2}$ Theoretical Physics Division, PINSTECH, P.O. Nilore, Islamabad 44000, Pakistan \\ ${ }^{3}$ Department of Physics, University of Azad Jammu and Kashmir Muzaffarabad, Azad Kashmir 13100, Pakistan \\ ${ }^{4}$ Department of Computer Sciences and Information Technology, University of Azad Kashmir, Azad Kashmir 13100, Pakistan
}

Correspondence should be addressed to Iftikhar Ahmad; aaiftikhar@yahoo.com

Received 18 January 2014; Revised 2 April 2014; Accepted 8 April 2014; Published 30 April 2014

Academic Editor: Ning $\mathrm{Hu}$

Copyright (C) 2014 Iftikhar Ahmad et al. This is an open access article distributed under the Creative Commons Attribution License, which permits unrestricted use, distribution, and reproduction in any medium, provided the original work is properly cited.

Radiation effects on magnetohydrodynamic (MHD) boundary-layer flow and heat transfer characteristic through a porous medium due to an exponentially stretching sheet have been studied. Formulation of the problem is based upon the variable thermal conductivity. The heat transfer analysis is carried out for both prescribed surface temperature (PST) and prescribed heat flux (PHF) cases. The developed system of nonlinear coupled partial differential equations is transformed to nonlinear coupled ordinary differential equations by using similarity transformations. The series solutions for the transformed of the transformed flow and heat transfer problem were constructed by homotopy analysis method (HAM). The obtained results are analyzed under the influence of various physical parameters.

\section{Introduction}

Since the revolutionary work of Sakiadis $[1,2]$ on boundarylayer flow past a moving surface a great deal of research work has been carried out for the two-dimensional boundary-layer flows. Crane [3] extended the Sakiadis [1, 2] flow problem by assuming a stretching boundary. Crane's problem is one of the flow problems in boundary layer theory that possesses an exact solution. The stretching velocity in Crane's problem is linearly proportional to the distance from origin. The heat and mass transfer on the flow past a porous stretching surface is discussed by P. S. Gupta and A. S. Gupta [4]. Brady and Acrivos [5] proved the existence and uniqueness of the solution for the stretching flow. Three-dimensional flow due to a stretching surface was analyzed by McLeod and Rajagopal [6]. In another paper Wang [7] extended Crane's problem for a stretching cylinder. Flow problems due to a stretching surface have important applications in technology, geothermal energy recovery, and manufacturing process such as oil recovery, artificial fibers, metal extrusion, and metal spinning. Extensive literature is available regarding the steady flows in Newtonian and non-Newtonian fluid flows over a stretching sheet. The readers are referred to the studies [8-14] and references therein. The transportation of heat in a porous medium has applications in geothermal systems, rough oil mining, soil-water contamination, and biomechanical problems. Vajravelu [15] reported steady flow and heat transfer of viscous fluids by considering different heating process in a porous medium.

The above mentioned problems deal with linear stretching of the surface. Magyari and Keller [16] initiated the work by assuming an exponentially stretching surface. The heat transfer analysis for flow past an exponentially stretching surface was carried out by Elbashbeshy [17]. The same problem by considering the constitutive equation of a viscoelastic fluid was investigated by Khan [18]. Due to the applications in electrical power generator, astrophysical flows, solar power technology, space vehicle reentry, and so forth, the heat transfer analysis in the presence of radiation is another important area of research. Raptis [19] investigated the radiation effects for the flow past a semi-infinite flat plate. The influence of thermal radiation in a viscoelastic fluid past a stretching sheet 
is illustrated by Chen [20]. Sajid and Hayat [21] discussed the homotopy series solution for the influence of radiation on the flow past an exponentially stretching sheet. Chiam [22] investigated the heat transfer analysis with variable thermal conductivity in a stagnation point flow towards a stretching sheet. In another paper, the analysis of effects of variable thermal conductivity was discussed by Chiam [23].

The purpose of the present paper is to demonstrate an analysis for the MHD flow and heat transfer analysis of a viscous fluid towards an exponentially stretching sheet in the presence of radiation effects and Darcy's resistance. The analytic series solution is presented by using homotopy analysis method [24-30].

\section{Mathematical Formulation of the Problem}

Consider a steady, incompressible, two-dimensional MHD flow of an electrically conducting viscous fluid towards an exponentially stretching sheet in a porous medium. The fluid occupies the space $y>0$ and is flowing in the $x$ direction and $y$-axis is normal to the flow. A constant magnetic field of strength $B_{0}$ is applied in the normal direction and the induced magnetic field is neglected which is a valid assumption when the magnetic Reynolds number is small. The boundary-layer equations that govern the present flow and heat transfer problem are

$$
\begin{gathered}
\frac{\partial u}{\partial x}+\frac{\partial v}{\partial y}=0 \\
u \frac{\partial u}{\partial x}+v \frac{\partial u}{\partial y}=-\frac{1}{\rho} \frac{\partial p}{\partial x}+\nu \frac{\partial^{2} u}{\partial y^{2}}-\frac{v}{k^{\prime}} u-\frac{\sigma B_{0}^{2}}{\rho} u \\
\frac{\partial p}{\partial y}=0 \\
\rho c_{p}\left(u \frac{\partial T}{\partial x}+v \frac{\partial T}{\partial y}\right)=\frac{\partial}{\partial y}\left(k \frac{\partial T}{\partial y}\right)-\frac{\partial q_{r}}{\partial y}
\end{gathered}
$$

where $u$ and $v$ are the components of velocity in $x$ and $y$ directions, respectively, $p$ is the pressure, $\rho$ is the density, $v$ represents kinematic viscosity, $k^{\prime}$ is the permeability of porous medium, $\sigma$ is the electrical conductivity, $T$ is the fluid temperature, $c_{p}$ is the specific heat, $q_{r}$ is radiative heat flux, and $k$ is the variable thermal conductivity defined in the following way:

$$
k= \begin{cases}k_{\infty}[1+\epsilon \theta(\eta)] & \text { in PST case } \\ k_{\infty}[1+\epsilon \phi(\eta)] & \text { in PHF case }\end{cases}
$$

in which $\epsilon$ is the small parameter $(\epsilon>0$ for gases and $\epsilon<0$ for solids and liquids) and $\theta(\eta)$ and $\phi(\eta)$ are dimensionless temperature distributions for PST and PHF cases, respectively. The relevant boundary conditions for the present problem are

$$
\begin{gathered}
u=U_{0} e^{x / l}, \quad v=0, \\
T=T_{\infty}+A e^{a x / 2 l}(\mathrm{PST}), \\
-k \frac{\partial T}{\partial y}=B e^{(b+l) x / 2 l}(\mathrm{PHF}), \quad \text { at } y=0, \\
u \longrightarrow 0, \quad T \longrightarrow T_{\infty} \quad \text { as } y \longrightarrow \infty,
\end{gathered}
$$

where $U_{0}$ is a characteristic velocity, $l$ is the characteristic length scale, and $A, a, B$, and $b$ are the parameters of temperature profiles depending on the properties of the liquid. Equation (7) suggests that $\partial p / \partial x=0$ far away from the plate. Since there is no variation in the pressure in $y$ direction as evident from (3), therefore, $\partial p / \partial x=0$ is zero throughout the flow field and we have from (2)

$$
u \frac{\partial u}{\partial x}+v \frac{\partial u}{\partial y}=\nu \frac{\partial^{2} u}{\partial y^{2}}-\frac{v}{k^{\prime}} u-\frac{\sigma B_{0}^{2}}{\rho} u .
$$

The radiative heat flux is defined as

$$
q_{r}=-\frac{4 \sigma_{1}}{3 m} \frac{\partial T^{4}}{\partial y}
$$

in which $\sigma_{1}$ is the Stefan-Boltzmann constant and $m$ is the coefficient of mean absorption. Assuming $T^{4}$ as a linear combination of temperature, then

$$
T^{4} \cong-3 T_{\infty}^{4}+4 T_{\infty}^{3} T \text {. }
$$

Substituting (9) and (10) in (4) one gets

$$
\rho c_{p}\left(u \frac{\partial T}{\partial x}+v \frac{\partial T}{\partial y}\right)=\frac{\partial}{\partial y}\left(k \frac{\partial T}{\partial y}\right)+\frac{16 T_{\infty}^{3} \sigma_{1}}{3 m} \frac{\partial^{2} T}{\partial y^{2}} .
$$

Introducing the dimensionless variables

$$
\begin{aligned}
& \eta=\sqrt{\frac{U_{0}}{2 v l}} e^{x / 2 l} y, \quad \psi(x, y)=\sqrt{2 v l U_{0}} e^{x / 2 l} f(\eta) . \\
& \theta(\eta)=\frac{T-T_{\infty}}{T_{w}-T_{\infty}}(\mathrm{PST}), \quad \phi(\eta)=\frac{T-T_{\infty}}{T_{w}-T_{\infty}}(\mathrm{PHF}) .
\end{aligned}
$$

In terms of above variables flow and heat transfer problems take the following form:

$$
\begin{array}{cc}
f^{\prime \prime \prime}-2 f^{\prime 2}+f f^{\prime \prime}-2(R+M) f^{\prime}=0, & \\
(1+\operatorname{Tr}+\epsilon \theta) \theta^{\prime \prime}+\operatorname{Pr} f \theta^{\prime}-a \operatorname{Pr} f^{\prime} \theta+\epsilon \theta^{\prime 2}=0 & (\mathrm{PST}), \\
(1+\operatorname{Tr}+\epsilon \phi) \phi^{\prime \prime}+\operatorname{Pr} f \phi^{\prime}-b \operatorname{Pr} f^{\prime} \phi+\epsilon \phi^{\prime 2}=0 & (\mathrm{PHF}), \\
f(0)=0, \quad f^{\prime}(0)=1, & \\
\theta(0)=1, \quad \phi^{\prime}(0)=-\frac{1}{1+\epsilon}, & \\
f^{\prime}(\infty)=\theta(\infty)=\phi(\infty)=0,
\end{array}
$$


where the dimensionless parameters are given as

$$
\begin{gathered}
R=\frac{\nu \varphi l}{k^{\prime} U_{0}} e^{-x / l}, \quad \operatorname{Pr}=\frac{\mu c_{p}}{k_{\infty}}, \\
M=\frac{l \sigma B_{\circ}^{2}}{\rho U_{0}} e^{-x / l}, \quad \operatorname{Tr}=\frac{16 \sigma_{1} T_{\infty}^{3}}{3 k_{\infty} K} .
\end{gathered}
$$

\section{HAM Solution}

To find the series solutions for velocity and temperature profiles given in (14), we use homotopy analysis method (HAM). The velocity and temperature distributions can be expressed in terms of the following base functions:

$$
\left\{\eta^{j} \exp (-n \eta) \mid j \geq 0, n \geq 0\right\}
$$

in the form

$$
\begin{gathered}
f(\eta)=a_{0,0}^{0}+\sum_{n=0}^{\infty} \sum_{k=0}^{\infty} a_{m, n}^{k} \eta^{k} \exp (-n \eta), \\
\theta(\eta)=\sum_{n=0}^{\infty} \sum_{k=0}^{\infty} b_{m, n}^{k} \eta^{k} \exp (-n \eta), \\
\phi(\eta)=\sum_{n=0}^{\infty} \sum_{k=0}^{\infty} c_{m, n}^{k} \eta^{k} \exp (-n \eta),
\end{gathered}
$$

where $a_{m, n}^{k}, b_{m, n}^{k}$, and $c_{m, n}^{k}$ are the coefficients. The following initial guesses are selected for solution expressions of $f(\eta)$, $\theta(\eta)$, and $\phi(\eta)$

$$
\begin{gathered}
f_{0}(\eta)=1-e^{-\eta}, \quad \theta_{0}(\eta)=e^{-\eta}, \\
\phi_{0}(\eta)=\frac{1}{1+\epsilon} e^{-\eta},
\end{gathered}
$$

and auxiliary linear operators are

$$
\begin{gathered}
\mathscr{L}_{1}(f)=f^{\prime \prime}-f^{\prime}, \quad \mathscr{L}_{2}(\theta)=\theta^{\prime \prime}-\theta, \\
\mathscr{L}_{3}(\phi)=\phi^{\prime \prime}-\phi,
\end{gathered}
$$

which satisfy

$$
\begin{gathered}
\mathscr{L}_{1}\left[C_{1}+C_{2} e^{\eta}+C_{3} e^{-\eta}\right]=0, \quad \mathscr{L}_{2}\left[C_{4} e^{\eta}+C_{5} e^{-\eta}\right]=0, \\
\mathscr{L}_{3}\left[C_{6} e^{\eta}+C_{7} e^{-\eta}\right]=0,
\end{gathered}
$$

where $C_{i}(i=1,2, \ldots, 7)$ are arbitrary constants. The zeroorder deformation problems are

$$
\begin{gathered}
(1-p) \mathscr{L}_{1}\left[\widehat{f}(\eta, p)-f_{0}(\eta)\right]=p \hbar_{f} \mathcal{N}_{f}[\widehat{f}(\eta, p)], \\
(1-p) \mathscr{L}_{2}\left[\widehat{\theta}(\eta, p)-\theta_{0}(\eta)\right]=p \hbar_{\theta} \mathcal{N}_{\theta}[\widehat{f}(\eta, p)], \\
(1-p) \mathscr{L}_{3}\left[\widehat{\phi}(\eta, p)-\phi_{0}(\eta)\right]=p \hbar_{\phi} \mathcal{N}_{\phi}[\widehat{f}(\eta, p)], \\
\widehat{f}(0, p)=0, \quad \widehat{f}^{\prime}(0, p)=1, \\
\widehat{\theta}(0, p)=1, \quad \frac{\partial \widehat{\phi}(0, p)}{\partial \eta}=-\frac{1}{1+\epsilon}, \\
\hat{f}^{\prime}(\infty, p)=\widehat{\theta}(\infty, p)=\widehat{\phi}(\infty, p)=0 .
\end{gathered}
$$

The nonlinear operators are given by

$$
\begin{aligned}
& \mathcal{N}_{f}[\widehat{f}(\eta, p)]=\frac{\partial^{3} \widehat{f}(\eta, p)}{\partial \eta^{3}}-2\left(\frac{\partial \widehat{f}(\eta, p)}{\partial \eta}\right)^{2} \\
& +\widehat{f}(\eta, p) \frac{\partial^{2} \widehat{f}(\eta, p)}{\partial \eta^{2}}-2(R+M) \frac{\partial \widehat{f}(\eta, p)}{\partial \eta}, \\
& \mathcal{N}_{\theta}[\widehat{f}(\eta, p), \widehat{\theta}(\eta, p)] \\
& =\left[\begin{array}{c}
\{1+\operatorname{Tr}+\epsilon \widehat{\theta}(\eta, p)\} \frac{\partial^{2} \hat{\theta}(\eta, p)}{\partial \eta^{2}}+\operatorname{Pr} \widehat{f}(\eta, p) \frac{\partial \hat{\theta}(\eta, p)}{\partial \eta} \\
-a \operatorname{Pr} \frac{\partial \widehat{f}(\eta, p)}{\partial \eta} \hat{\theta}(\eta, p)+\epsilon\left(\frac{\partial \widehat{\theta}(\eta, p)}{\partial \eta}\right)^{2}
\end{array}\right], \\
& =\left[\begin{array}{c}
\{1+\operatorname{Tr}+\epsilon \widehat{\phi}(\eta, p)\} \frac{\partial^{2} \widehat{\phi}(\eta, p)}{\partial \eta^{2}}+\operatorname{Pr} \widehat{f}(\eta, p) \frac{\partial \widehat{\phi}(\eta, p)}{\partial \eta} \\
-b \operatorname{Pr} \frac{\partial \widehat{f}(\eta, p)}{\partial \eta} \widehat{\phi}(\eta, p)+\epsilon\left(\frac{\partial \hat{\phi}(\eta, p)}{\partial \eta}\right)^{2}
\end{array}\right],
\end{aligned}
$$

in which $p \in[0,1]$ is an embedding parameter and $\hbar_{f}, \hbar_{\theta}$, and $\hbar_{\phi}$ are nonzero auxiliary parameters. For $p=0$ and $p=1$, we, respectively, have

$$
\begin{array}{ll}
\widehat{f}(\eta, 0)=f_{0}(\eta), & \widehat{f}(\eta, 1)=f(\eta), \\
\hat{\theta}(\eta, 0)=\theta_{0}(\eta), & \widehat{\theta}(\eta, 1)=\theta(\eta), \\
\widehat{\phi}(\eta, 0)=\phi_{0}(\eta), & \widehat{\phi}(\eta, 1)=\phi(\eta) .
\end{array}
$$


As $p$ varies from 0 to $1, \widehat{f}(\eta, p), \widehat{\theta}(\eta, p)$, and $\widehat{\phi}(\eta, p)$ vary from $f_{0}(\eta), \theta_{0}(\eta)$, and $\phi_{0}(\eta)$ to $f(\eta), \theta(\eta)$, and $\phi(\eta)$, respectively. Using Taylor's series one can write

$$
\begin{aligned}
& \widehat{f}(\eta, p)=f_{0}(\eta)+\sum_{m=1}^{\infty} f_{m}(\eta) p^{m}, \\
& \widehat{\theta}(\eta, p)=\theta_{0}(\eta)+\sum_{m=1}^{\infty} \theta_{m}(\eta) p^{m}, \\
& \widehat{\phi}(\eta, p)=\phi_{0}(\eta)+\sum_{m=1}^{\infty} \phi_{m}(\eta) p^{m},
\end{aligned}
$$

where

$$
\begin{aligned}
& f_{m}(\eta)=\left.\frac{1}{m !} \frac{\partial^{m} f(\eta, p)}{\partial p^{m}}\right|_{p=0}, \\
& \theta_{m}(\eta)=\left.\frac{1}{m !} \frac{\partial^{m} \theta(\eta, p)}{\partial p^{m}}\right|_{p=0}, \\
& \phi_{m}(\eta)=\left.\frac{1}{m !} \frac{\partial^{m} \phi(\eta, p)}{\partial p^{m}}\right|_{p=0} .
\end{aligned}
$$

Note that the zero-order deformation equations contain three auxiliary parameters $\hbar_{f}, \hbar_{\theta}$, and $\hbar_{\phi}$. The convergence of series solutions strongly depends upon these three parameters. Assuming $\hbar_{f}, \hbar_{\theta}$, and $\hbar_{\phi}$ are chosen in such a way that the above series are convergent at $p=1$, then

$$
\begin{aligned}
& f(\eta)=f_{0}(\eta)+\sum_{m=1}^{\infty} f_{m}(\eta), \\
& \theta(\eta)=\theta_{0}(\eta)+\sum_{m=1}^{\infty} \theta_{m}(\eta), \\
& \phi(\eta)=\phi_{0}(\eta)+\sum_{m=1}^{\infty} \phi_{m}(\eta) .
\end{aligned}
$$

Taking the derivative of (21)-(24) $m$-times with respect to $p$, then selecting $p=0$ and dividing by $m$ ! the following expressions for $m$ th order deformation problems are obtained:

$$
\begin{gathered}
\mathscr{L}_{1}\left[f_{m}(\eta)-\chi_{m} f_{m-1}(\eta)\right]=\hbar_{f} \mathfrak{N}_{m}^{f}(\eta), \\
\mathscr{L}_{2}\left[\theta_{m}(\eta)-\chi_{m} \theta_{m-1}(\eta)\right]=\hbar_{\theta} \mathfrak{N}_{m}^{\theta}(\eta), \\
\mathscr{L}_{3}\left[\phi_{m}(\eta)-\chi_{m} \phi_{m-1}(\eta)\right]=\hbar_{\phi} \mathfrak{N}_{m}^{\phi}(\eta), \\
f_{m}(0)=f_{m}^{\prime}(0)=f_{m}^{\prime}(\infty)=\theta_{m}(0)=\theta_{m}(\infty) \\
=\phi_{m}^{\prime}(0)=\phi_{m}(\infty)=0,
\end{gathered}
$$

in which

$$
\begin{gathered}
\mathfrak{N}_{m}^{f}(\eta)=f_{m-1}^{\prime \prime \prime}-2(R+M) f_{m-1}^{\prime} \\
+\sum_{k=0}^{m-1}\left[f_{m-1-k} f_{k}^{\prime \prime}-2 f_{m-1-k}^{\prime} f_{k}^{\prime}\right], \\
\mathfrak{N}_{m}^{\theta}(\eta)=(1+\operatorname{Tr}) \theta_{m-1}^{\prime \prime}+\sum_{k=0}^{m-1}\left[\epsilon \theta_{m-1-k} \theta_{k}^{\prime \prime}+\operatorname{Pr} f_{m-1-k} \theta_{k}^{\prime}\right. \\
\left.\quad-a \operatorname{Pr} f_{m-1-k}^{\prime} \theta_{k}+\epsilon \theta_{m-1-k}^{\prime} \theta_{k}^{\prime}\right], \\
\mathfrak{N}_{m}^{\phi}(\eta)=(1+\operatorname{Tr}) \phi_{m-1}^{\prime \prime}+\sum_{k=0}^{m-1}\left[\epsilon \phi_{m-1-k} \phi_{k}^{\prime \prime}+\operatorname{Pr} f_{m-1-k} \phi_{k}^{\prime}\right. \\
\left.-b \operatorname{Pr} f_{m-1-k}^{\prime} \phi_{k}+\epsilon \phi_{m-1-k}^{\prime} \phi_{k}^{\prime}\right],
\end{gathered}
$$

The system of linear nonhomogeneous equations (29)-(32) is solved using Mathematica in the order $m=1,2,3, \ldots$.

\section{Results and Discussion}

The governing nonlinear equations for the MHD flow and heat transfer analysis for a viscous fluid in a porous medium with radiation effects and variable thermal conductivity are solved analytically using homotopy analysis method for both PST and PHF cases. The solutions are given in the form of analytic expressions in (28). These analytic expressions contain the auxiliary parameters $\hbar_{f}, \hbar_{\theta}$, and $\hbar_{\phi}$. The convergence and rate of approximation of the obtained series solutions strongly depend upon these parameters. To examine the range of acceptable values of these parameters, we draw the $\hbar$ curves in Figure 1 for the functions $f(\eta), \theta(\eta)$, and $\phi(\eta)$. This figure depicts that the admissible values of $\hbar_{f}, \hbar_{\theta}$, and $\hbar_{\phi}$ are in the interval $[-0.6,-0.3]$.

To see the influence of porosity parameter $R$, Prandtl number Pr, thermal conductivity $\epsilon$, temperature parameters $a$ and $b$, and thermal radiation $T r$ on the temperature distributions for both PST and PHF cases, Figures 2-6 have been plotted. Figures (a) are for the PST case and Figures (b) are for PHF case. In Figures 2(a) and 2(b), variation of temperature distributions with variation in thermal conductivity parameter $\epsilon$ is shown. Figure 2(a) illustrates that temperature and thermal boundary-layer thickness in the PST case increases by increasing thermal conductivity. The results in the PHF case are opposite to that of PST case and are shown in Figure 2(b). Figure 3 elucidates that the temperature and thermal boundary-layer thickness increases by an increase in the porosity parameter. Since in the governing equation magnetic parameter and porosity parameter appear in the same way, therefore, the effects of magnetic parameter are similar to those of porosity parameter. The behavior of temperature with changing Prandtl number is quite opposite to that of 


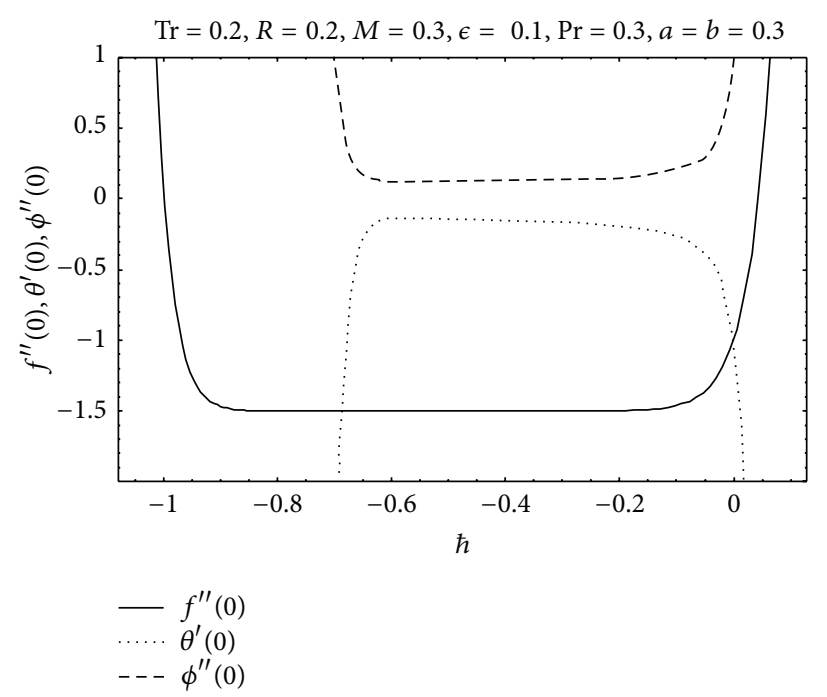

FIGURE 1: $\hbar$-curves for the functions $f(\eta), \theta(\eta)$, and $\phi(\eta)$ at 20th-order of approximation.

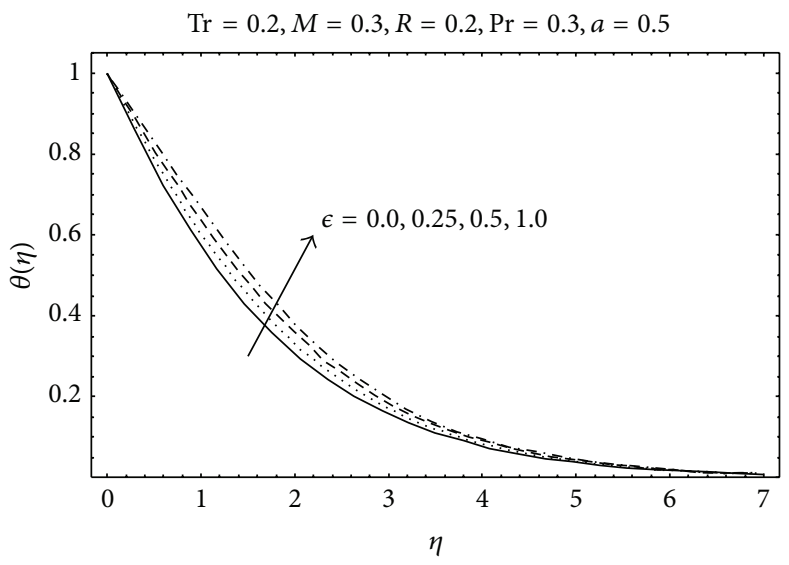

(a)

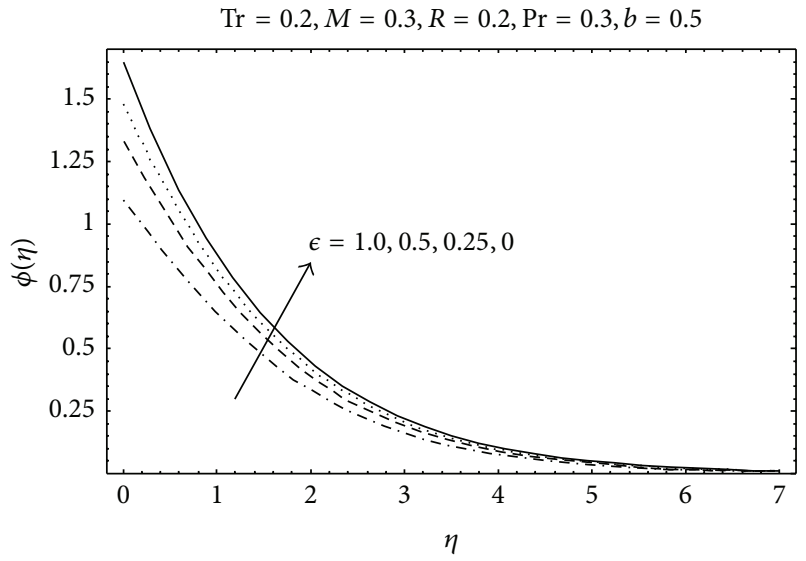

(b)

FIGURE 2: Influence of thermal conductivity parameter $\epsilon$ on temperature profiles (a) for PST case and (b) for PHF case.

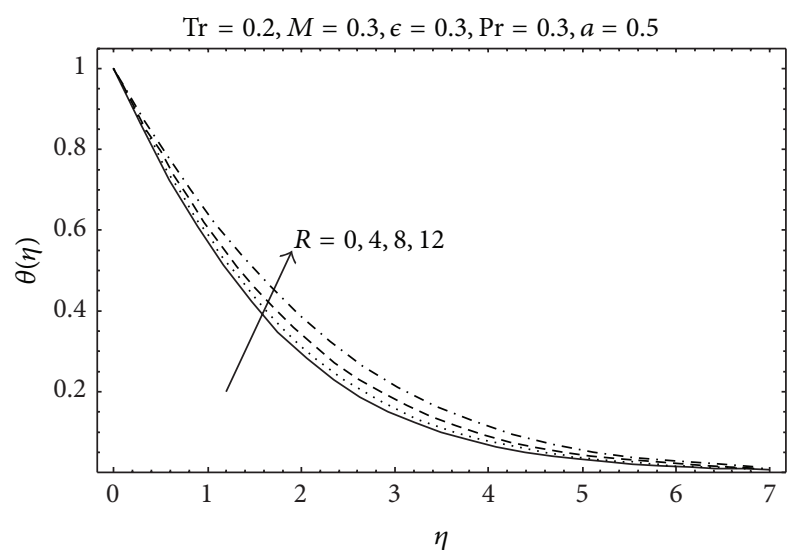

(a)

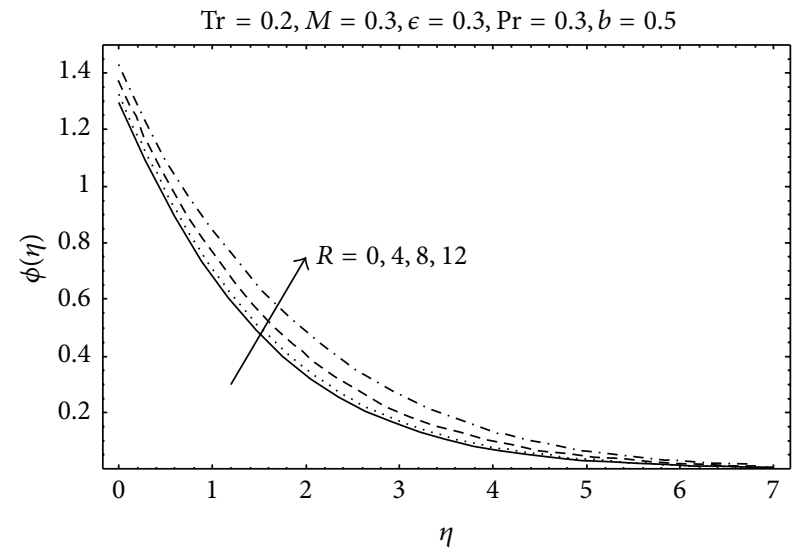

(b)

FIGURE 3: Influence of porosity parameter $R$ on temperature profiles (a) for PST case and (b) for PHF case. 


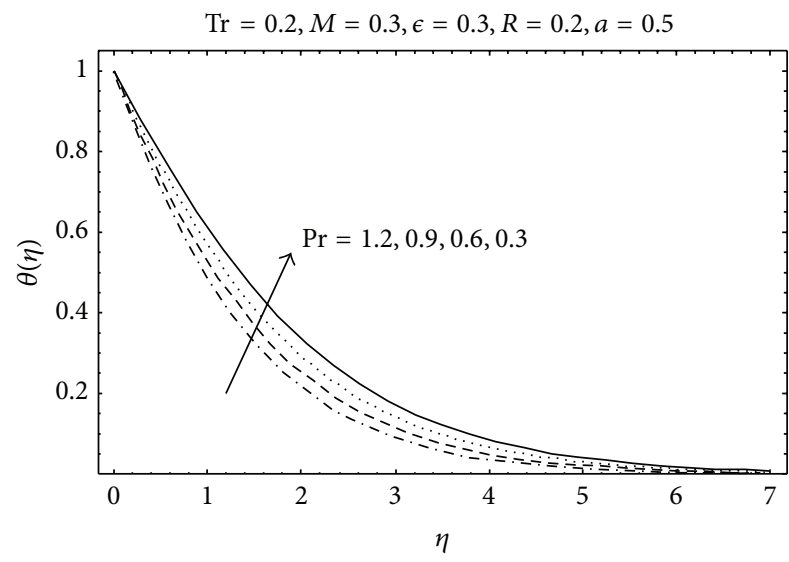

(a)

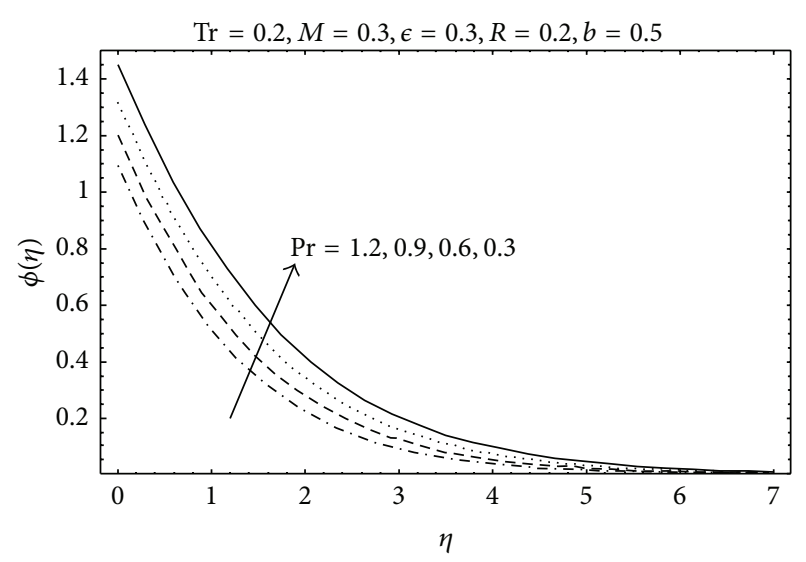

(b)

FIGURe 4: Influence of Prandtl number Pr on temperature profiles (a) for PST case and (b) for PHF case.

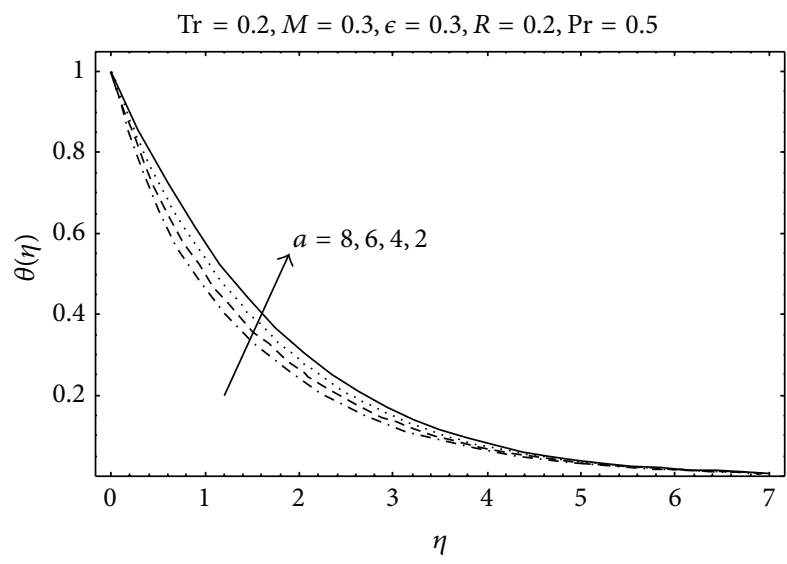

(a)

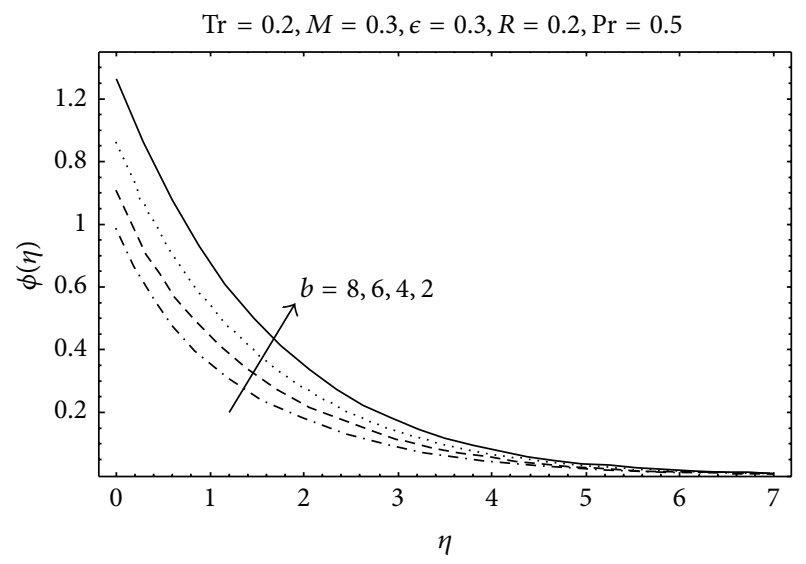

(b)

FIGURE 5: Influence of parameters $a$ and $b$ on temperature profiles (a) for PST case and (b) for PHF case.

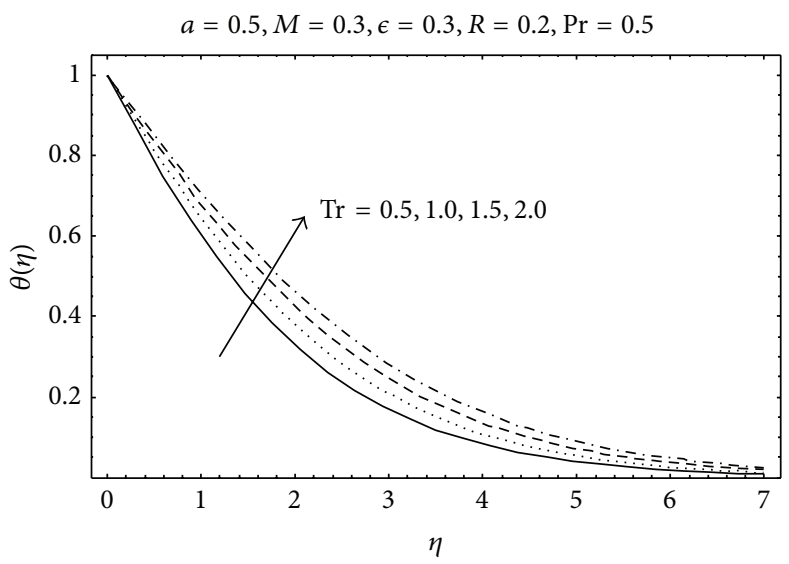

(a)

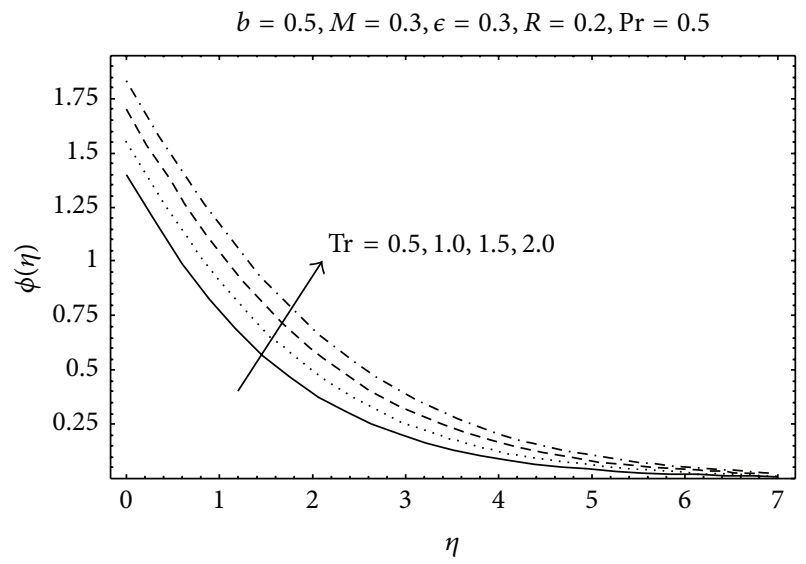

(b)

FIgURE 6: Influence of thermal radiation parameter Tr on temperature profiles (a) for PST case and (b) for PHF case. 
porosity parameter and is given in Figure 4. Figure 5 is made to show the influence of parameter $a$ in the PST case and parameter $b$ in the PHF case on the temperature distributions. It is evident from Figure 5 that effects of these parameters are the same as that of Prandtl number. The influence of thermal radiation on the temperature profiles for both PST and PHF cases is depicted in Figure 6. The plotted profiles show that the temperature and thermal boundary-layer thickness is an increasing function of the thermal radiation.

\section{Conclusions}

Magnetohydrodynamic boundary-layer flow and heat transfer characteristic due to exponentially stretching sheet in the presence of thermal radiation, Darcy resistance, and variable thermal conductivity have been studied in the paper. The similarity solutions are developed using homotopy analysis method. Following are the findings of current study.

(1) The variable thermal conductivity has an impact in enhancing the temperatures profile in PST case and reduction in temperature in PHF case.

(2) The effect of porosity parameter $R$ increases the temperature in both PST and PHF cases.

(3) The effect of increasing the values of Prandtl number is to decrease the thermal boundary-layer thickness in both of the cases.

\section{Conflict of Interests}

The authors declare that there is no conflict of interests regarding the publication of this paper.

\section{Acknowledgment}

Muhammad Sajid acknowledges the support from AS-ICTP.

\section{References}

[1] B. C. Sakiadis, "Boundary layer behaviour on continuous solid surfaces," AIChE Journal, vol. 7, no. 1, pp. 26-28, 1961.

[2] B. C. Sakiadis, "Boundary layer behaviour on continuous solid surfaces: II, the boundary layer on a continuous flat surface," AIChE Journal, vol. 17, pp. 221-225, 1961.

[3] L. J. Crane, "Flow past a stretching plate," Zeitschrift für Angewandte Mathematik und Physik, vol. 21, no. 4, pp. 645-647, 1970.

[4] P. S. Gupta and A. S. Gupta, "Heat and mass transfer on a stretching sheet with suction and blowing," The Canadian Journal of Chemical Engineering, vol. 55, no. 6, pp. 744-746, 1977.

[5] J. F. Brady and A. Acrivos, "Steady flow in a channel or tube with an accelerating surface velocity. An exact solution to the NavierStokes equations with reverse flow," Journal of Fluid Mechanics, vol. 112, pp. 127-150, 1981.

[6] J. B. McLeod and K. R. Rajagopal, "On the uniqueness of flow of a Navier-Stokes fluid due to a stretching boundary," Archive for Rational Mechanics and Analysis, vol. 98, no. 4, pp. 385-393, 1987.

[7] C. Y. Wang, "The three-dimensional flow due to a stretching flat surface," Physics of Fluids, vol. 27, no. 8, pp. 1915-1917, 1984.
[8] C. Y. Wang, "Fluid flow due to a stretching cylinder," Physics of Fluids, vol. 31, pp. 466-468, 1988.

[9] R. Cortell, "Flow and heat transfer of a fluid through a porous medium over a stretching surface with internal heat generation/absorption and suction/blowing," Fluid Dynamics Research, vol. 37, no. 4, pp. 231-245, 2005.

[10] R. Cortell, "A note on magnetohydrodynamic flow of a powerlaw fluid over a stretching sheet," Applied Mathematics and Computation, vol. 168, no. 1, pp. 557-566, 2005.

[11] S.-J. Liao, "On the analytic solution of magnetohydrodynamic flows of Non-Newtonian fluids over a stretching sheet," Journal of Fluid Mechanics, no. 488, pp. 189-212, 2003.

[12] P. D. Ariel, T. Hayat, and S. Asghar, "The flow of an elastico-viscous fluid past a stretching sheet with partial slip," Acta Mechanica, vol. 187, no. 1-4, pp. 29-35, 2006.

[13] P. D. Ariel, "Axisymmetric flow of a second grade fluid past a stretching sheet," International Journal of Engineering Science, vol. 39, no. 5, pp. 529-553, 2001.

[14] T. Hayat and M. Sajid, "Analytic solution for axisymmetric flow and heat transfer of a second grade fluid past a stretching sheet," International Journal of Heat and Mass Transfer, vol. 50, no. 1-2, pp. 75-84, 2007.

[15] K. Vajravelu, "Flow and heat transfer in a saturated porous medium," Zeitschrift für angewandte Mathematik und Mechanik, vol. 74, pp. 605-614, 1994.

[16] E. Magyari and B. Keller, "Heat and mass transfer in the boundary layers on an exponentially stretching continuous surface," Journal of Physics D: Applied Physics, vol. 32, no. 5, pp. 577-585, 1999.

[17] E. M. A. Elbashbeshy, "Heat transfer over an exponentially stretching continuous surface with suction," Archives of Mechanics, vol. 53, no. 6, pp. 643-651, 2001.

[18] S. K. Khan, "Boundary layer vicoelastic flow over an exponential stretching sheet," International Journal of Applied Mechanics and Engineering, vol. 11, pp. 321-335, 2006.

[19] A. Raptis, C. Perdikis, and H. S. Takhar, "Effect of thermal radiation on MHD flow," Applied Mathematics and Computation, vol. 153, no. 3, pp. 645-649, 2004.

[20] C.-H. Chen, "On the analytic solution of MHD flow and heat transfer for two types of viscoelastic fluid over a stretching sheet with energy dissipation, internal heat source and thermal radiation," International Journal of Heat and Mass Transfer, vol. 53, no. 19-20, pp. 4264-4273, 2010.

[21] M. Sajid and T. Hayat, "Influence of thermal radiation on the boundary layer flow due to an exponentially stretching sheet," International Communications in Heat and Mass Transfer, vol. 35, no. 3, pp. 347-356, 2008.

[22] T. C. Chiam, "Heat transfer with variable conductivity in a stagnation-point flow towards a stretching sheet," International Communications in Heat and Mass Transfer, vol. 23, no. 2, pp. 239-248, 1996.

[23] T. C. Chiam, "Heat transfer in a fluid with variable thermal conductivity over a linearly stretching sheet," Acta Mechanica, vol. 129, no. 1-2, pp. 63-72, 1998.

[24] S. J. Liao, The proposed homotopy anslysis technique for the solution of non-linear problems [Ph.D. thesis], Shanghai Jiao Tong University, 1992.

[25] S. J. Liao, Beyond Perturbation: Introduction to Homotopy Analysis Method, Chapman \& Hall/CRC, Boca Raton, Fla, USA, 2003. 
[26] S. Liao, "A new branch of solutions of boundary-layer flows over an impermeable stretched plate," International Journal of Heat and Mass Transfer, vol. 48, no. 12, pp. 2529-2539, 2005.

[27] S. J. Liao, "An analytic solution of unsteady boundary-layer flows caused by an impulsively stretching plate," Communications in Nonlinear Science and Numerical Simulation, vol. 11, no. 3, pp. 326-339, 2006.

[28] M. Sajid, T. Hayat, and S. Asghar, "On the analytic solution of the steady flow of a fourth grade fluid," Physics Letters A: General, Atomic and Solid State Physics, vol. 355, no. 1, pp. 18-26, 2006.

[29] Z. Abbas, M. Sajid, and T. Hayat, "MHD boundary-layer flow of an upper-convected Maxwell fluid in a porous channel," Theoretical and Computational Fluid Dynamics, vol. 20, no. 4, pp. 229-238, 2006.

[30] S. Abbasbandy, "The application of homotopy analysis method to nonlinear equations arising in heat transfer," Physics Letters A, vol. 360, no. 1, pp. 109-113, 2006. 


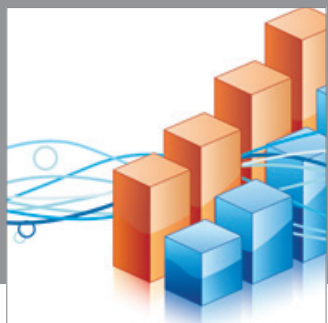

Advances in

Operations Research

mansans

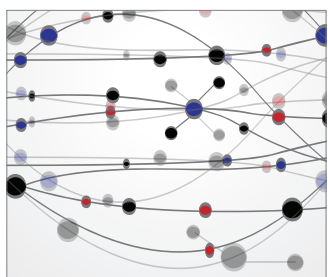

The Scientific World Journal
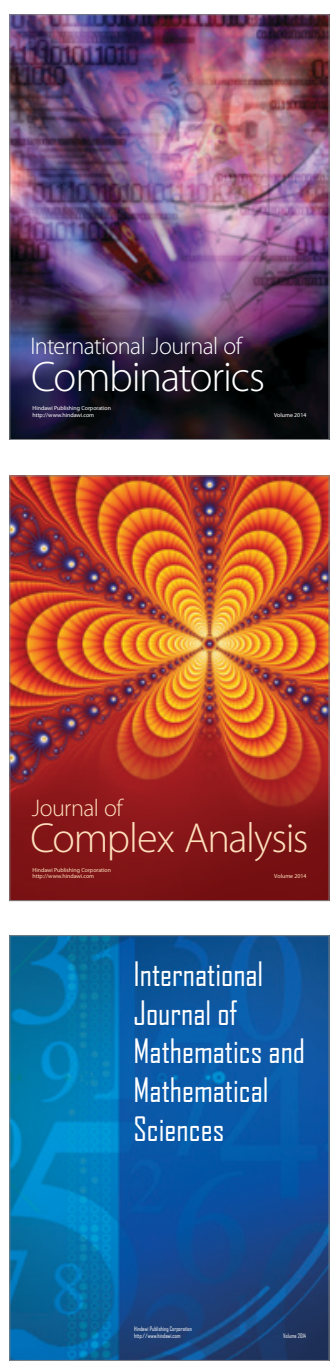
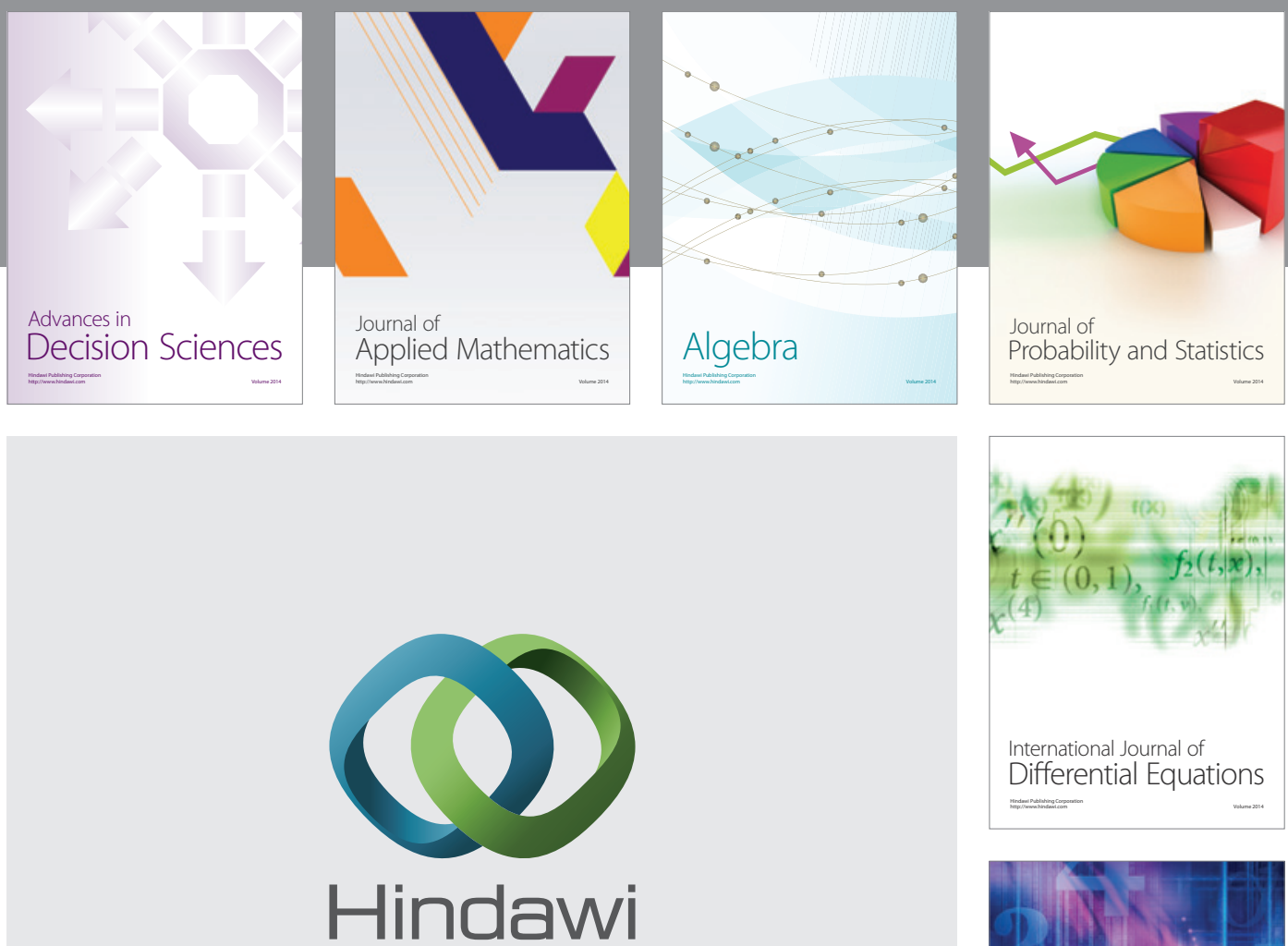

Submit your manuscripts at http://www.hindawi.com
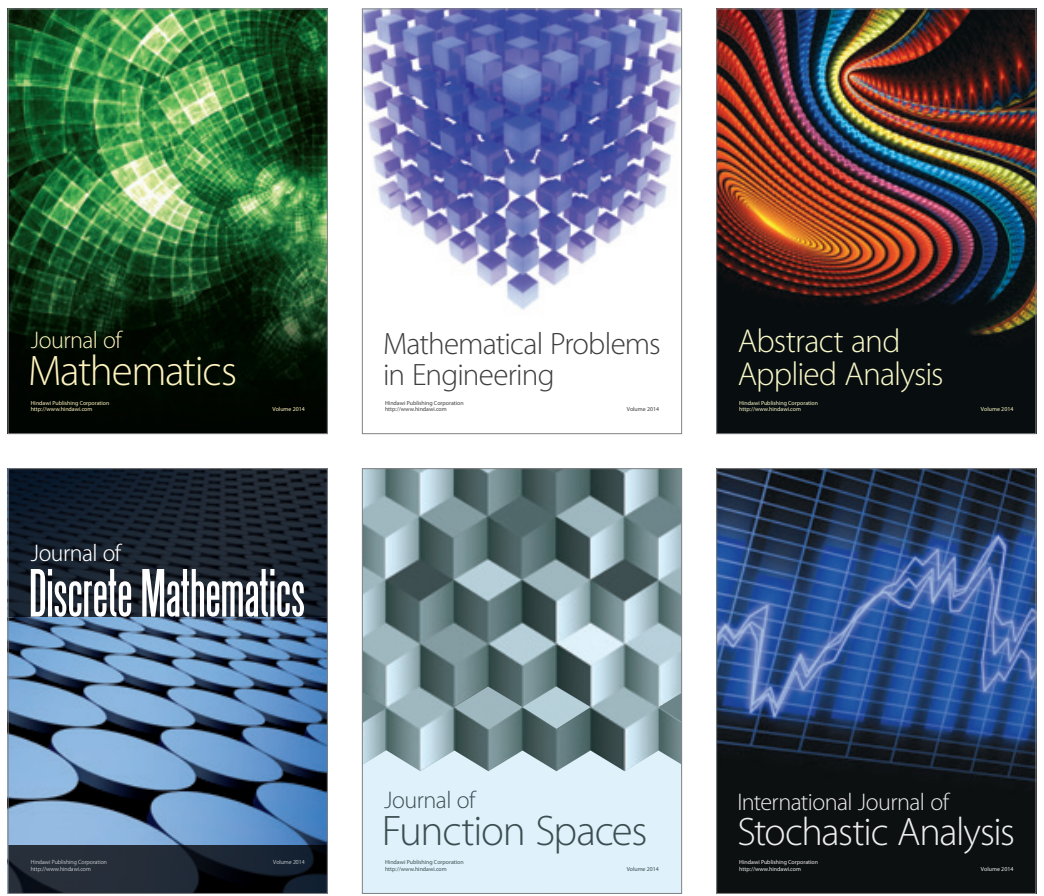

Journal of

Function Spaces

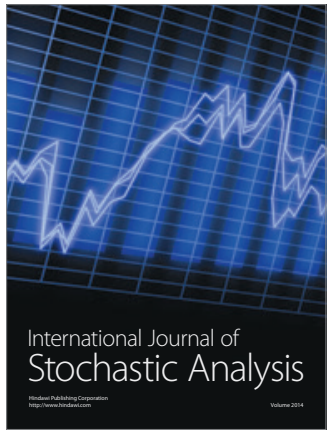

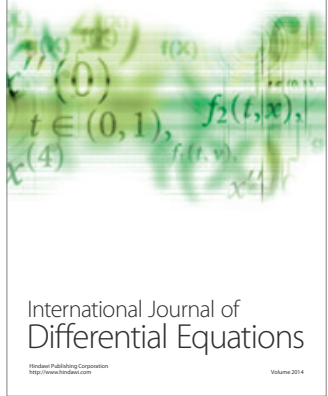
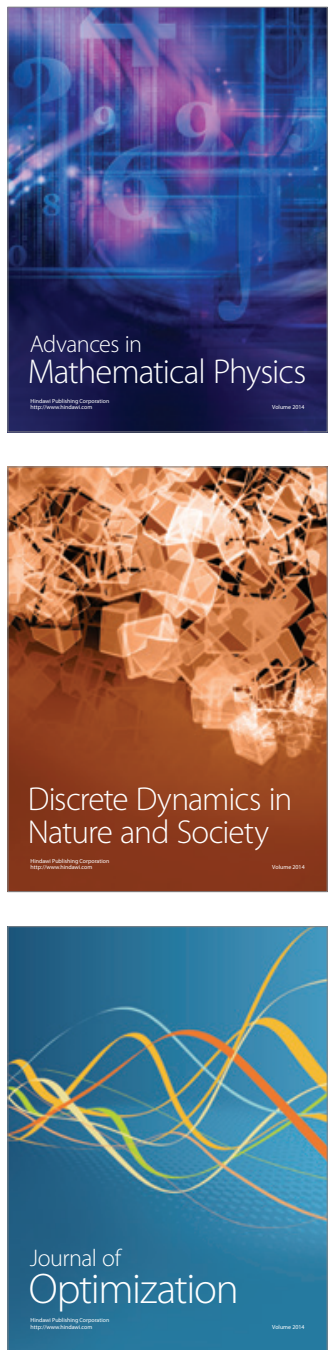